\title{
A Simple Power Source Modeling and Experimental Investigation of a Spacecraft for EMC Applications
}

\author{
Tae-Youn $\mathrm{Kim}^{1,2} \cdot \mathrm{Jae}-\mathrm{Hyun} \mathrm{Lee}^{2, *}$
}

\begin{abstract}
Simplified modeling of a spacecraft power system is proposed to predict the conducted electromagnetic compatibility (EMC) environment. A key element of the spacecraft conducted environment is the electrical power subsystem, which corresponds to the power source. Since the impedance of the power source has a significant effect on conducted interference, the assessment of power bus impedance is essential to control and manage spacecraft EMC. In the proposed model, all components inside the power system were modeled as simple equivalent circuits with lumped elements. The advantage is that it simplifies the complex power system so that its impedance can be quickly obtained at the early stage of spacecraft system design and development. The model is validated by comparing the calculated results with experimental data. The comparison results between simulation and experiment showed good agreements.
\end{abstract}

Key Words: Battery, Bus Impedance, Conducted Emission, Conducted Susceptibility, Electrical Test Bed, EMC, PCDU, Spacecraft Power System.

\section{INTRODUCTION}

In the spacecraft development process, the system and units are designed and manufactured according to electromagnetic compatibility (EMC) requirements defined at the start of the project. In the integration phase of the flight model system, only qualified units can be installed in accordance with EMC requirements. In the final step, system-level EMC testing is performed to ensure that EMC requirements are met. Since EMC requirements have a significant impact on the entire spacecraft development process described above, it is vital to evaluate and analyze the spacecraft at the beginning of the project to ensure it has sufficient margin with the EMC requirements. It is possible to request a design change of the spacecraft unit earlier or redefine the EMC requirement based on the analysis results. This reduces the risk of EMC test failures at the equipment and system levels and avoids costly remedies and time-consuming redesigns [1].

The spacecraft's EMC environment can be divided into a conducted environment through cables and a radiated environment through free space. This paper focuses on the conducted environment. A key element of the spacecraft conducted environment is the electrical power subsystem (EPS), which corresponds to the power source. Since the power source impedance has a significant effect on conducted interference, impedance control is essential for the management of conducted emission (CE) and susceptibility (CS) of spacecraft units. Therefore, the power impedance envelope is specified in the EMC requirements, and all units are tested based on this impedance envelope. Thus, modeling for EPS is of paramount importance in evaluat-

Manuscript received September 9, 2020 ; Revised November 5, 2020 ; Accepted December 17, 2020. (ID No. 20200909-138J)

${ }^{1}$ Space Environment Test Division, Korea Aerospace Research Institute, Daejeon, Korea.

${ }^{2}$ Department of Radio and Information Communications Engineering, Chungnam National University, Daejeon, Korea.

"Corresponding Author: Jae-Hyun Lee (e-mail: jaehyun@cnu.ac.kr)

This is an Open-Access article distributed under the terms of the Creative Commons Attribution Non-Commercial License (http://creativecommons.org/licenses/by-nc/4.0) which permits unrestricted non-commercial use, distribution, and reproduction in any medium, provided the original work is properly cited.

(c) Copyright The Korean Institute of Electromagnetic Engineering and Science. All Rights Reserved. 
ing whether the spacecraft design meets EMC requirements. This can be a powerful tool to ensure a system-conducted EMC safety margin, such as implementing an appropriate bus filter or tailoring the unit's CS limit in the early design stage of a spacecraft [2]. Research has been performed to characterize the function of the electrical power subsystem $[3,4]$. However, the previous researches have analyzed only the power system's functional performance, not the EMC characteristics.

Until now, a heritage- or experience-based approach has been applied to EMC analysis without an appropriate model. Conducted EMC analysis of spacecraft has not been done on analytical methods through modeling. From this perspective, this paper proposes a simple approach to the modeling of spacecraft power sources for conducted EMC analysis. The model is validated by comparing the calculated results with experimental data. This simplified method, which uses an equivalent circuit model, can help system engineers quickly evaluate spacecraft EMC performance early in spacecraft development. This paper is organized as follows. Section II explains the modeling methodology and shows the equivalent circuit model of the spacecraft power system. Section III describes the experimental configuration, and Section IV discusses the verification results of the proposed model by comparing the impedance through the power bus model with the experimentally obtained bus impedance characteristics.

\section{SPACECRAFT POWER SYSTEM MODELING}

The noise coupling process in the spacecraft conducted environment is illustrated in Fig. 1. The CE current noise generated from the unit is combined with the power bus impedance and then induces a voltage ripple at the power interface. This system-level voltage becomes the input noise coupled to each unit in the real spacecraft system. When comparing this noise with the unit's CS verification level, the system can be considered electromagnetically compatible if there is a positive margin.

This process is called the conducted EMC safety margin

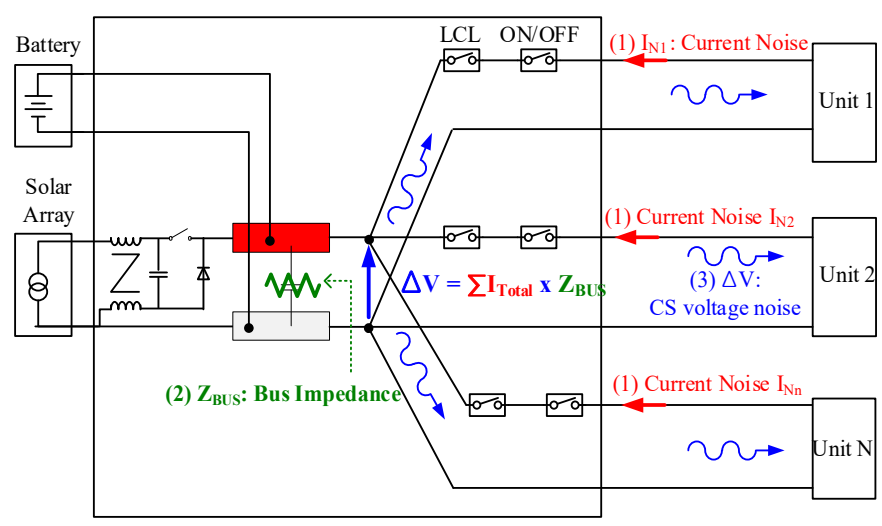

Fig. 1. Conducted EMI coupling in the spacecraft system.

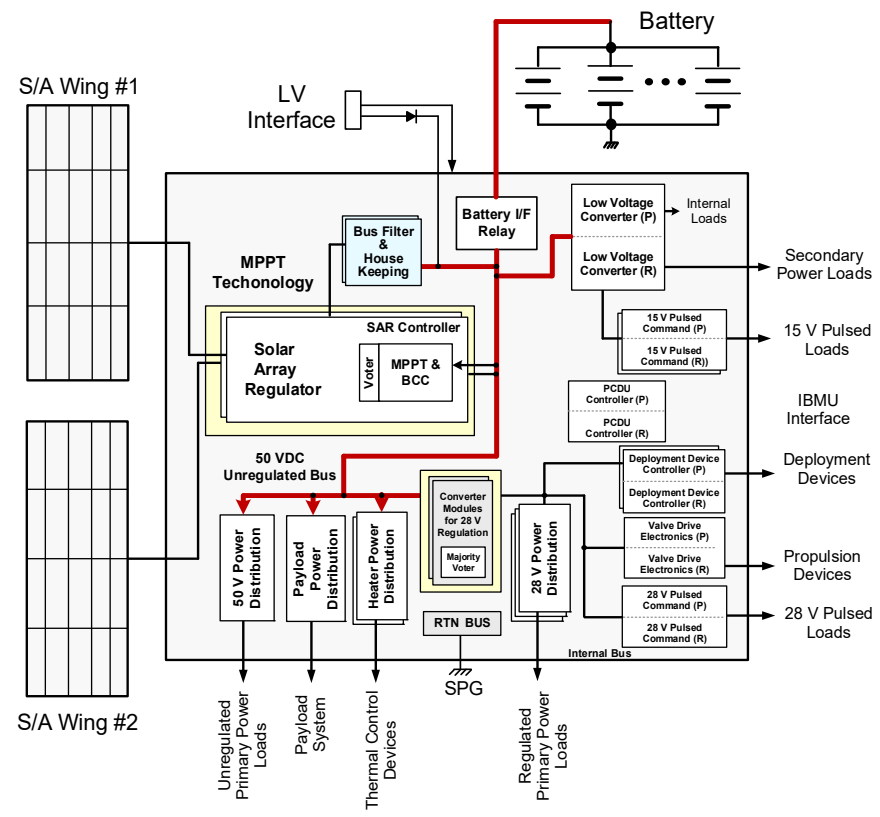

Fig. 2. Block diagram of the spacecraft electrical power system.

analysis of the spacecraft. The factors that determine the conducted EMC safety margin are (1) unit CE test levels, (2) unit CS test levels, and (3) primary power bus impedance. In this paper, a modeling method to obtain the power bus impedance of the spacecraft is proposed.

The power bus impedance can be analyzed by modeling the spacecraft EPS. The typical EPS consists of a solar array, an energy storage system (i.e., battery), and a power control and distribution unit (PCDU), as shown in Fig. 2.

The solar array converts solar energy into electrical energy, and it is delivered to the loads and battery. The power generated by the solar array panel is controlled and converted through the solar array regulator (SAR) of the PCDU. The central distribution and protection of converted power are carried out by the bus filter and power distribution board (PDB) of the PCDU. The battery is directly connected to the DC power bus to provide power during eclipses or periods when the bus load exceeds the solar array's power capability. Although many modeling methods have been studied to analyze only EPS performance, this paper proposes a simple analysis method that can easily grasp the impedance characteristics by expressing the spacecraft power system with the equivalent circuit model.

With regard to the system model, a modular concept approach was used. The factors that affect the power bus impedance are the battery and some parts of the PCDU. This power bus can be represented by a simple block diagram, as shown in Fig. 3.

\section{Battery Model}

Many battery models have been introduced and studied in 


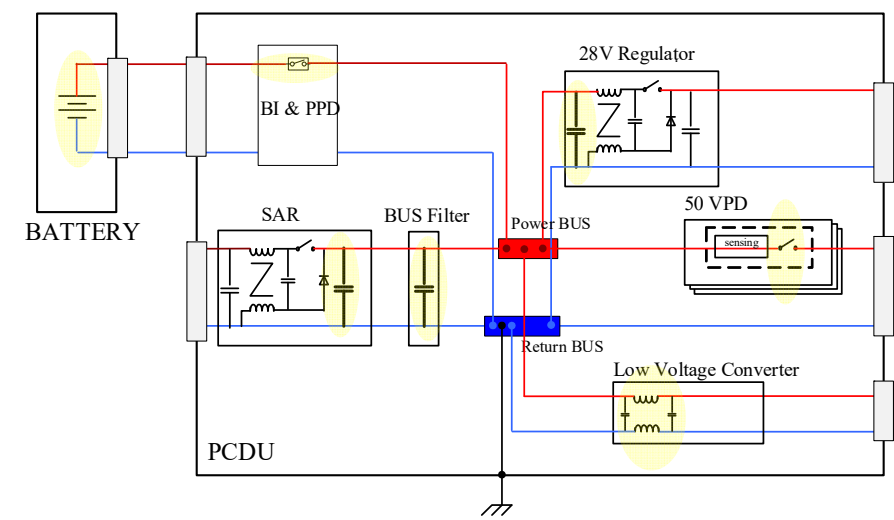

Fig. 3. Simplified block diagram of the spacecraft power bus.

various applications. These models can be classified as electrochemical, mathematical, electrical, and polynomial [5]. Waag et al. [6] investigated battery impedance at various conditions and aging states. Mesbahi et al. [5] studied the dynamic model of lithium-ion batteries incorporating electrothermal and aging aspects. Wu et al. [7] presented the equivalent $\mathrm{AC}$ impedance model for the electrochemical process between the anode and cathode. Tabbakh et al. [8] investigated battery modeling, including electrochemical impedance spectroscopy (EIS) and the charge/discharge dynamic modeling tests, and suggested a model including only inductances and resistances. Pelissou et al. [9] established an equivalent cell model through battery tests, especially EIS measurements.

Most studies have shown that battery impedance is highly dependent on battery conditions, such as state-of-charge (SoC), temperature, current rate, and lifetime. The simplified model illustrated in Fig. 4 is commonly accepted as an electrical equivalent circuit for the battery plate [10], and the same battery model was proposed in [7] and [9]. $L$ is the inductor linked to the electrode current collectors, $R$ is the series resistance of the cells, which mainly functions as temperature, $R_{c t}$ is called the charge transfer resistance, $C_{d l}$ is the double-layer capacitance of the electrode material/electrolyte interfaces [9].

However, the goal of battery modeling in this paper is to build a simple model using minimal information about the battery, such as voltage capacity and mechanical configuration.

In Fig. 4, some parameters are unknown and cannot be directly applicable to the battery. Accordingly, we have selected the battery model described in [11]. Fig. 5 shows the proposed

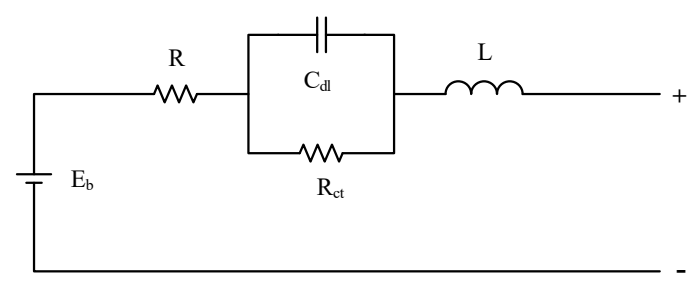

Fig. 4. Classic battery AC impedance model.

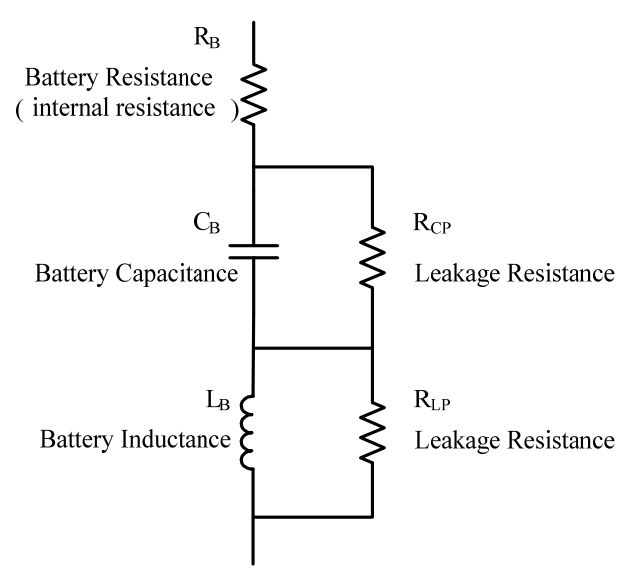

Fig. 5. Proposed equivalent circuit model for lithium-ion battery.

Table 1. Parameters for battery model

\begin{tabular}{ccc}
\hline & Description & Formula \\
\hline$L_{B}$ & Battery inductance & $L_{B}=\left(l_{C}[\mathrm{~cm}]+l_{W}[\mathrm{~cm}]\right) \times 10[\mathrm{nH} / \mathrm{cm}] \times \frac{N_{C S}}{N_{C P}}$ \\
$C_{B}$ & Battery capacitance & $C_{B}=0.23[\mathrm{~F} / \mathrm{Ah}] \times C_{C}[\mathrm{Ah}] \times \frac{N_{C P}}{N_{C S}}$ \\
$R_{B}$ & Battery resistance & $R_{B}=\left(R_{C}+R_{W}\right) \times \frac{N_{C S}}{N_{C P}}$ \\
$R_{L P}$ & Battery inductance & $R_{L P}=0.01 \pi \times\left(l_{C}[\mathrm{~cm}]+l_{W}[\mathrm{~cm}]\right) \times \frac{N_{C P}}{N_{C S}}$ \\
& parallel resistance & \\
$R_{C P}$ & Battery capacitance & $R_{C P}=0.05[\Omega A h] \times \frac{1}{C_{C}[\mathrm{Ah}]} \times \frac{N_{C S}}{N_{C P}}$ \\
\hline
\end{tabular}

where, $N_{C S}$ is the cell number connecting in series, $N_{C P}$ is the cell number connecting in parallel, $R_{C}$ is the internal resistance of cell, $R_{W}$ is the wire resistance, $C_{C}$ is the battery capacity, $l_{c}$ is the cell length, and $l_{w}$ is the wire length.

equivalent circuit model for the lithium-ion battery, and impedance parameters can be expressed by the formula in Table 1 .

The battery configuration in this model is $4 \mathrm{P} 13 \mathrm{~S}$ with VES100. Detailed parameters are as follows:

- Battery type: Lithium-ion

- Battery dimension: $855 \times 425 \times 210 \mathrm{~mm}^{3} \max$

- Battery capacity: 104 AH @ BOL

- Battery mass: $58 \mathrm{~kg}$

- Cell connection: 4 cells parallel and 13 cells serial connected

- Cell length: $5.4 \mathrm{~cm}$

Battery impedance measurements were compared to the simulations. The measured impedance of the battery is provided by the manufacturer. The comparison of the measured and proposed model results is depicted in Fig. 6. Results confirm that two impedances match very well up to a frequency of $10 \mathrm{kHz}$.

\section{Power Control and Distribution Unit Model}

The PCDU is in charge of receiving solar power from the solar array and regulating the battery charging current [12]. The 


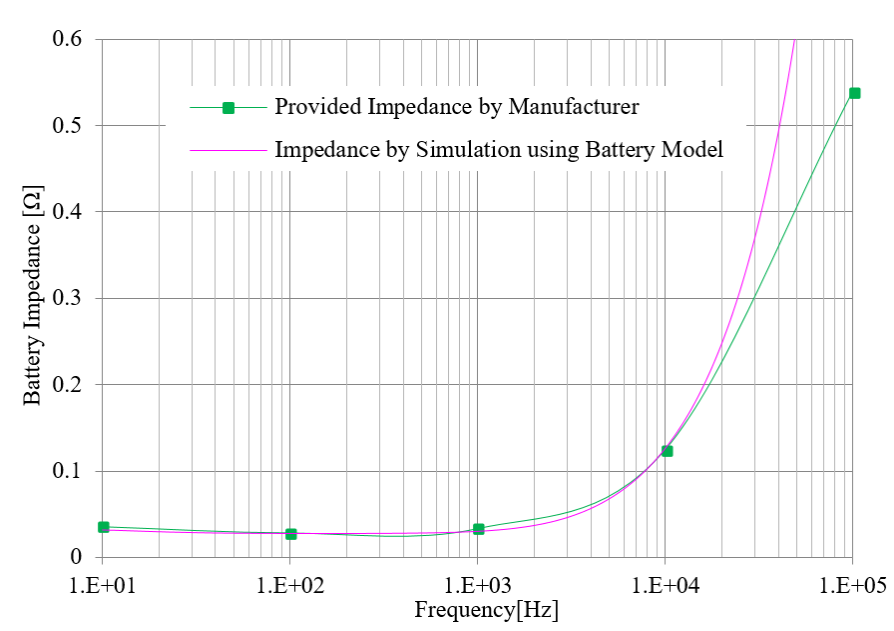

Fig. 6. Comparison of simulated impedance and provided impedance by the manufacturer for the battery.

structure of the PCDU power bus is shown in Fig. 7.

All modules are connected to the power bus, which is linked with the battery. The PCDU is composed of very complex pieces of electronics, but the following modules can be considered as factors that determine the impedance of the power bus in the PCDU:

- Solar array regulator,

- $28 \mathrm{~V}$ Power regulator,

- Low voltage converter,

- Bus filter,

- Battery interface,

- Power distribution module,

- Bus bar, board interconnection, internal wiring.

\subsection{Modeling of solar array regulator}

The SAR converts the solar array output voltage to the unregulated primary bus voltage $(50 \mathrm{~V})$. The SAR module consists of the buck power stage, maximum power point tracking (MPPT) block, driving block, protection block, and status monitoring (Fig. 8).

The output of the SAR is connected to the battery and power bus. The impedance of the SAR module on the power bus is dominated by the output filter installed on the output side of the regulator. The SAR consists of two boards with three regulators on each board, all working simultaneously under normal conditions. Thus, all six regulators are connected in parallel to the power bus. The total capacitance of the SAR consists of 18 capacitors connected in parallel with a $22 \mu \mathrm{F}$ capacitance. EUROFARAD's PM90 series capacitors are used in SAR output filters, and each regulator can be modeled as an ideal capacitor with the equivalent series inductance (ESL) and the equivalent series resistance (ESR). The parasitic inductance and resistance values of the PM90 capacitor are calculated using data provided by the manufacturer.

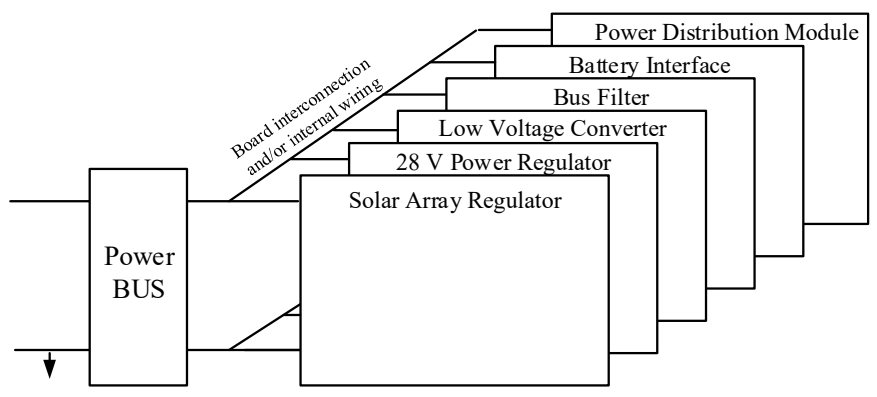

Fig. 7. Power bus interface inside the PCDU.

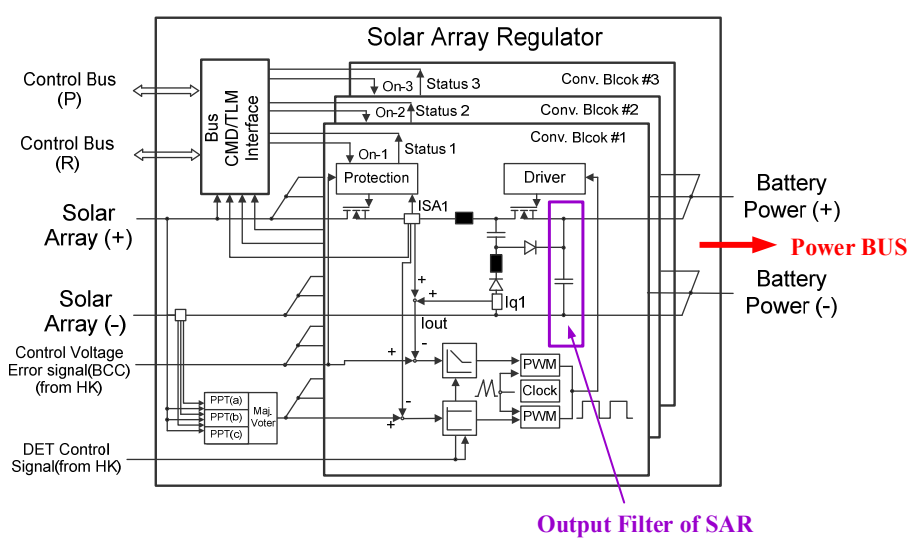

Fig. 8. Block diagram of solar array regulator in PCDU.

\subsection{Modeling of power $(28 \mathrm{~V}$ ) regulator}

The $28 \mathrm{~V}$ power regulator converts the unregulated primary power to a regulated power voltage. The input side of the $28 \mathrm{~V}$ power regulator is connected to the primary power bus, so the impedance model corresponds to the regulator input filter. There are two modules in the $28 \mathrm{~V}$ regulator, and each module consists of two converters. This means that four regulators are connected in parallel to the power bus. The impedance model of the $28 \mathrm{~V}$ regulator can be modeled as a capacitor installed at the input side of the converter, as shown in Fig. 9. The total capacitance of the $28 \mathrm{~V}$ power regulator has a configuration of 4 capacitors connected in parallel with a $10 \mu \mathrm{F}$ capacitance.

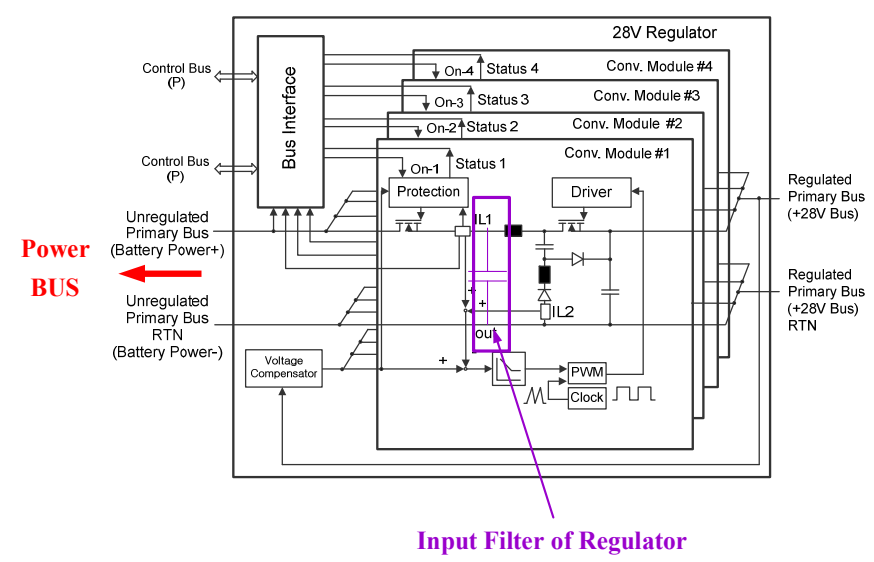

Fig. 9. Block diagram of $28 \mathrm{~V}$ power regulator in PCDU. 


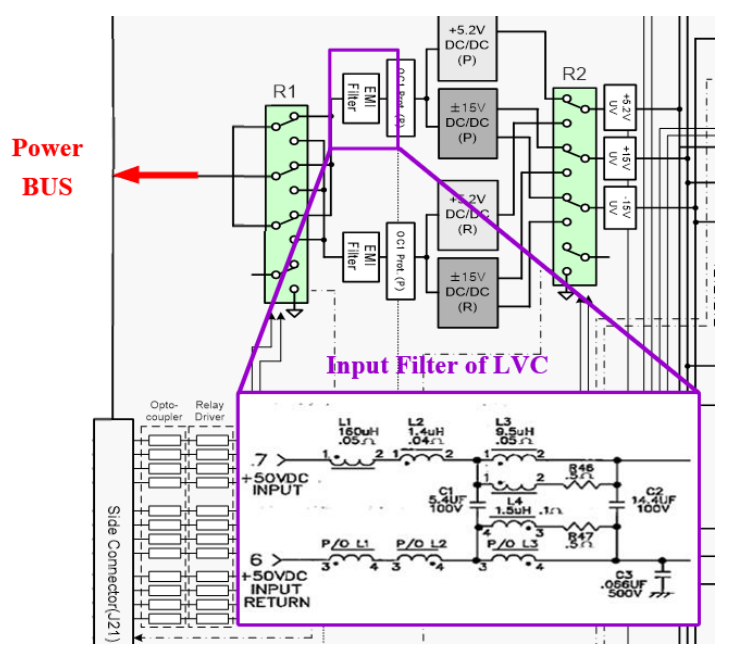

Fig. 10. Block diagram of low voltage converter in PCDU.

\subsection{Modeling of low voltage converters}

A low voltage converter (LVC) is the auxiliary power supply. It is powered by the unregulated primary power bus and generates a low voltage distributed to all other modules inside the PCDU. The input filter of the converter, such as the $28 \mathrm{~V}$ power regulator, is considered in the impedance model. Fig. 10 shows the block diagram of the low voltage converter. The battery impedance is mainly dominant at the low frequency, so neither the input filter model nor the input filter with the converter model affects the bus impedance. In the high-frequency range, all models have the same impedance characteristics. Therefore, only the input filter model is applied to the simple analysis.

\subsection{Modeling of the bus filter}

The bus filter module in the PCDU is responsible for the primary bus noise reduction function. It consists of a parallel assembly of metalized foil capacitors and is modeled by a capacitor circuit. The parasitic inductance and the equivalent resistance are expected to be very low due to parallel operation. The bus filter module is arranged in two boards with 16 capacitors on each board. The total capacitance of the bus filter has a configuration in which 32 capacitors with $33 \mu \mathrm{F}$ capacitance are connected in parallel.

\subsection{Modeling of battery interface and power distribution boards}

The battery interface module provides switching and protection devices to safely connect and disconnect the battery from the power system. It is modeled as the contact resistance of a mechanical relay. The PDB distribute the unregulated primary power to the payload and bus system on current-limiting protected output lines. Each power output line consists of a latching current limiter (LCL), so the PDB is represented by the internal resistance of the FET as a power switch.

\section{Connection Model between Battery and PCDU}

The connection of the battery to the PCDU is modeled by using a lumped parameter equivalent RLC circuit that includes contact resistance, taking into account the gauge, number, and length of wires. Potential parasitic effects such as internal harness inductance or interconnection resistance among modules were not considered in the power model. Since it is difficult to assess the power bus location during impedance measurement, the simulation point is defined as the power distribution location. It is represented by the PCDU internal wiring in Fig. 11.

\section{Proposed Power System Model}

Fig. 11 shows the resulting equivalent circuit model by combining the battery model and related elements of the PCDU. The novelty of the proposed model is to replace all components with an equivalent circuit of a capacitor because the power component's impedance mainly depends on the input and output filters (capacitors), which are directly connected to the power bus. In the proposed model, all components inside the battery and PCDU were modeled using minimal information provided by the manufacturer and then integrated. The advantage was to simplify the complex power subsystem so that its impedance could be quickly obtained.

\section{EXPERIMENTAL VERIFICATION}

Experimental measurement was done on the electrical test bed (ETB) platform to confirm the validity of the proposed model. The ETB is composed of engineering model (EM) units of the spacecraft and provides a test environment, which is required for acceptance tests at the system level, prior to flight model (FM) testing [13]. It is used to verify the electrical interface between each subsystem and the system-level functions, including the flight software. Fig. 12 shows the ETB platform, and as it is integrated into a flight-like electrical configuration, especially the PCDU, the proposed power source model can be applied to the ETB.

As illustrated in Fig. 13, the power bus impedance of the ETB platform can be verified in two ways: a CE test and a CS test.

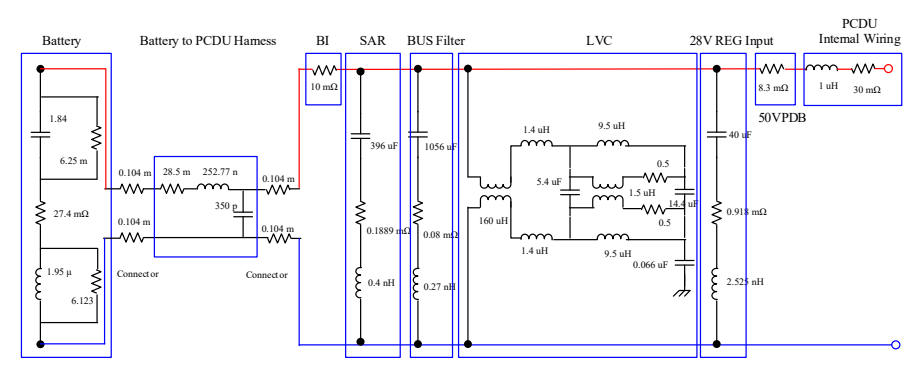

Fig. 11. Equivalent circuit model for the spacecraft power system (proposed model). 


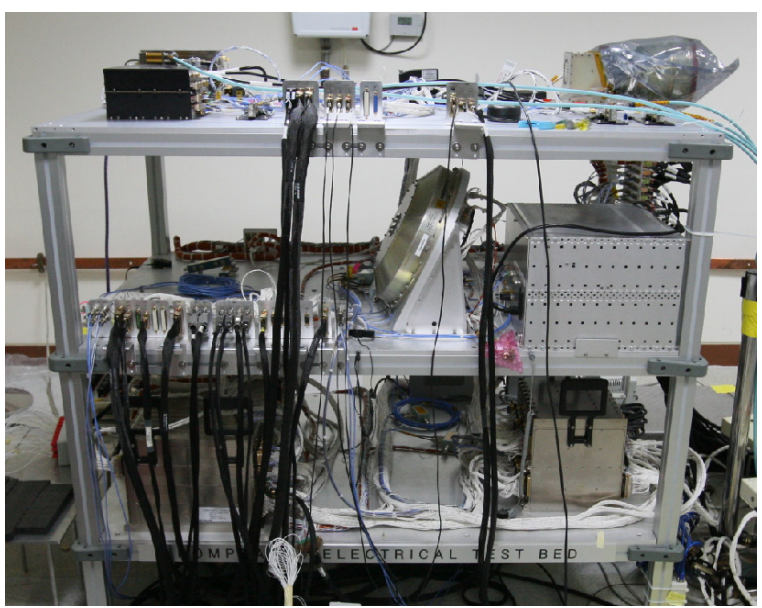

Fig. 12. Picture of an electrical test bed for spacecraft.

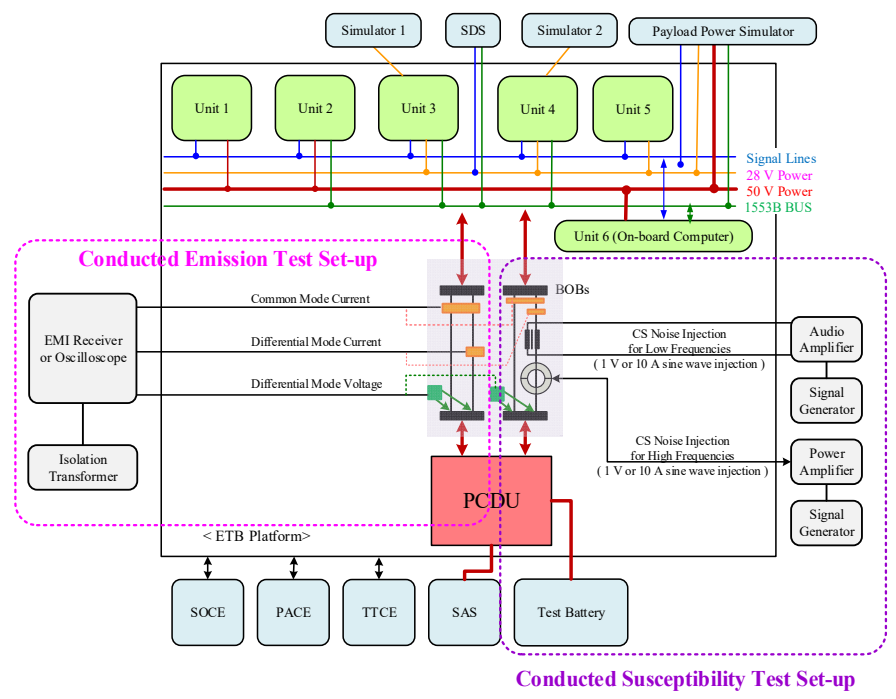

Fig. 13. Experimental setup for conducted emission and susceptibility test on ETB platform.

In the $\mathrm{CE}$ test, bus impedance can be deduced from the ratio of the voltage measured at the power distribution star point to the measured currents of all units connected on the power bus obtained in the frequency domain during the system energized in the operational mode. The bus impedance in a distribution point (i.e., a power star point) can be estimated by the following Eq. (1).

$$
Z_{\text {BUS }}=\frac{V_{\text {BUS_Star-Point }}}{\sqrt{\left(\left(I_{\text {Unit_A }}\right)^{2}+\left(I_{\text {Unit_B }}\right)^{2}+\left(I_{\text {Unit_C }}\right)^{2}+\cdots+\left(I_{\text {Unit_Z }}\right)^{2}\right)}}
$$

The CS test was carried out to confirm whether a given design of the spacecraft electrical system can withstand the sustained conducted noise. After injecting a small AC current $I_{\text {NOISE }}$ into the power bus using an independent current source, voltage perturbation $V_{\text {NOISE }}$ occurred according to its impedance characteristics. From these current and voltage results, the dynamic impedance of the power bus can be calculated.

The experimental setup for the CE and CS tests on the ETB

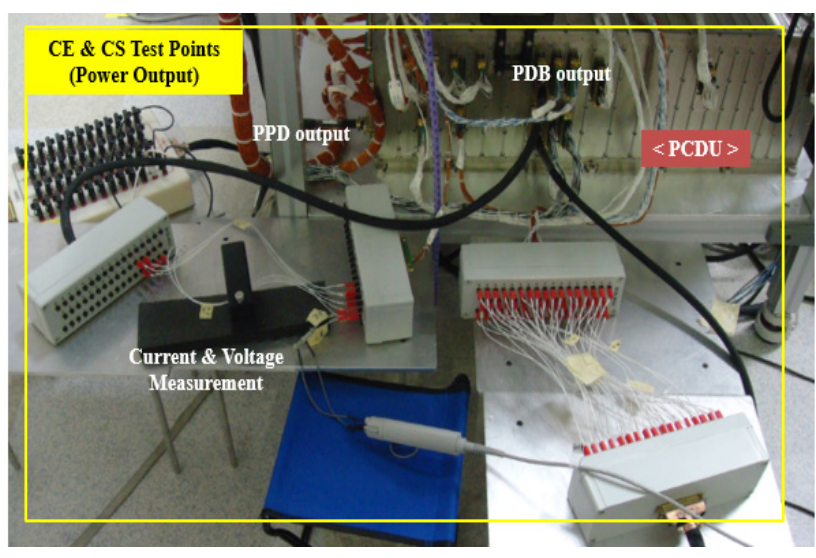

Fig. 14. Current and voltage measurement point; PPD output and PDB output of PCDU installed in ETB.

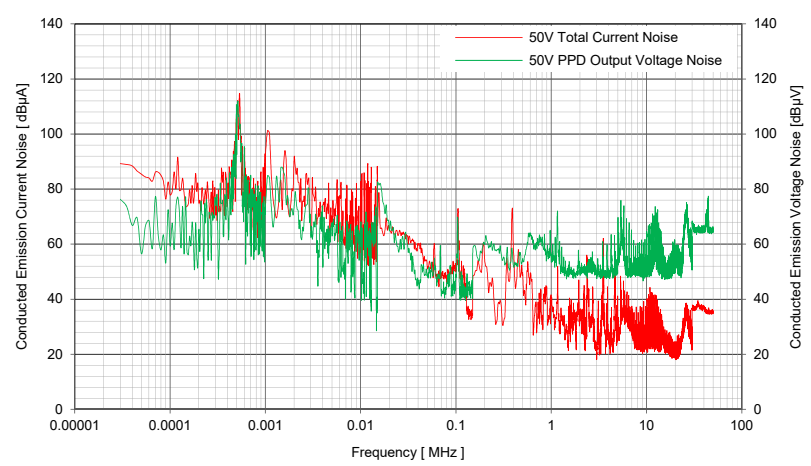

Fig. 15. Measured current and voltage emission in the $50 \mathrm{~V}$ power lines of the ETB platform.

platform is shown in Fig. 14. Test points are PCDU power outlets, which are $50 \mathrm{VPDB}$, and the payload power distribution (PPD) board. The PDB and PPD are connected to the same power bus $(50 \mathrm{~V})$, but the PPD is directly interfaced to the bus, and the PDB is connected through the LCL circuit. This means that the PPD output is the star point of the unregulated primary power bus. Fig. 15 shows the measurement results of the $\mathrm{CE}$ voltage and current on the ETB platform. The green line is the voltage noise of the $50 \mathrm{~V}$ power bus, and the red line is the total current noise of all $50 \mathrm{~V}$ units. These measurement data can be used to calculate the bus impedance on the ETB platform.

\section{RESULTS AND ANALYSIS}

A circuit simulator, such as PSPICE, was used to simulate the bus impedance with the proposed power bus model. An ETB CS test configuration consisting of a $50 \mathrm{~V}$ power load model and a noise injection model was added to the analysis model for comparison with the experimental results. The $50 \mathrm{~V}$ power load was modeled using a lumped $R L C$ network consisting of unit input filters, internal wiring, and unit function. The PPD output was connected to electrical ground support equip- 
ment (EGSE) made of resistors. Therefore, the PPD output was modeled as a resistor, taking power consumption into account.

Figs. 16 and 17 show the comparison of the measured impedance by the CS injection test and the simulated impedance using the proposed power system model for the PPD output and the PDB output, respectively. The CS test at the PPD output was performed up to the frequency range of $10 \mathrm{kHz}$, as shown in Fig. 16; thus, the impedance characteristics above 10 $\mathrm{kHz}$ could not be compared. However, since the measurement result increased from $4 \mathrm{kHz}$, which showed a similar trend with the simulation, the impedance characteristics after $10 \mathrm{kHz}$ can be expected to be similar to the analysis result. The comparison between the simulation and the measurement showed good agreement with little deviation. This deviation is caused by the difference between the actual measurement conditions and the analysis model, such as PPD output connected to the EGSE instead of the actual spacecraft unit. The lack of information from the EGSE made it difficult to implement a complete analysis model on the ETB platform.

Fig. 17 compares the measurement and analysis results for the real hardware of the ETB platform. In Fig. 17, the capacitive characteristics of simulated results are lower than that of test results. This can be caused by the ETB unit model. It is hardly possible to estimate all essential parasitic elements to create a detailed equivalent circuit of the test environment. Nevertheless, the results show a similar shape to the impedance curve.

To confirm the proposed model's validity, the impedance calculated from the $\mathrm{CE}$ test results was compared. Fig. 18 shows the comparison of the impedance deduced from the $\mathrm{CE}$ test results, as shown in Fig. 15, and the simulated impedance using the proposed power system model at the power star point. The measurement result is the root sum square of conducted current emission generated by 6 units installed in the ETB divided by the conducted voltage emission measured at the PPD output.

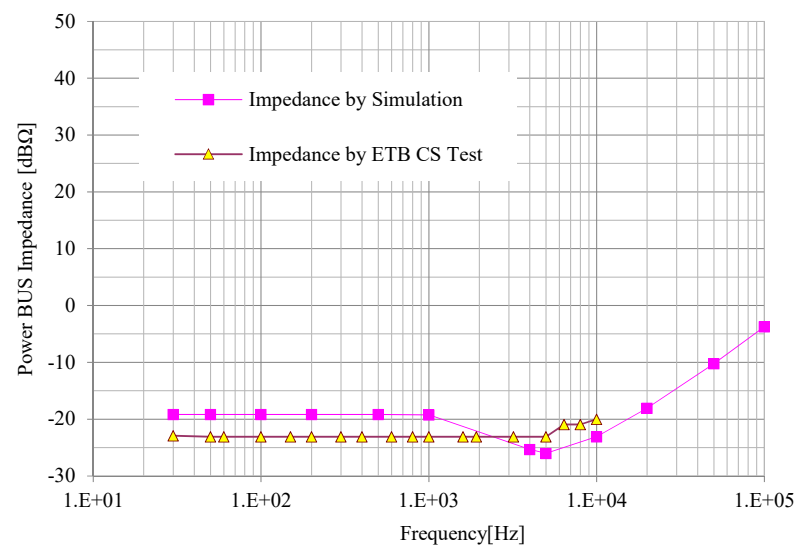

Fig. 16. Comparison of simulated impedance and measured impedance in ETB CS test environment for the PPD output point.

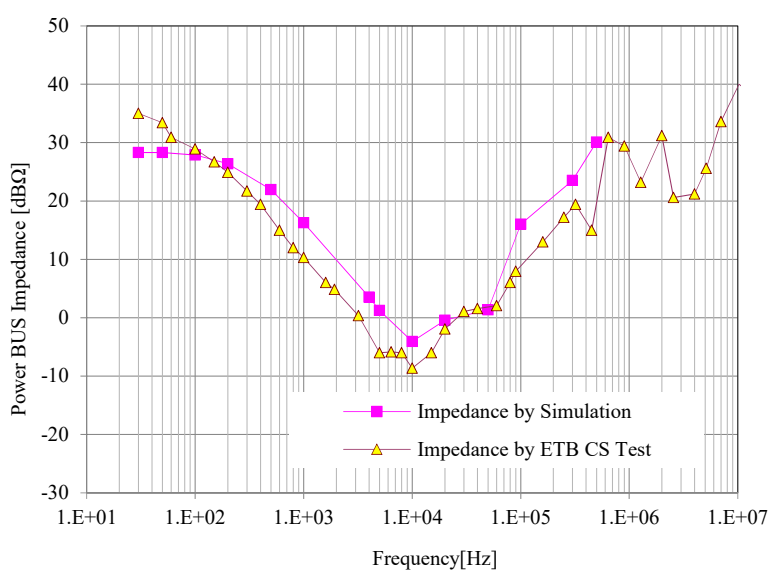

Fig. 17. Comparison of simulated impedance and measured impedance in ETB CS test environment for the PDB output point.

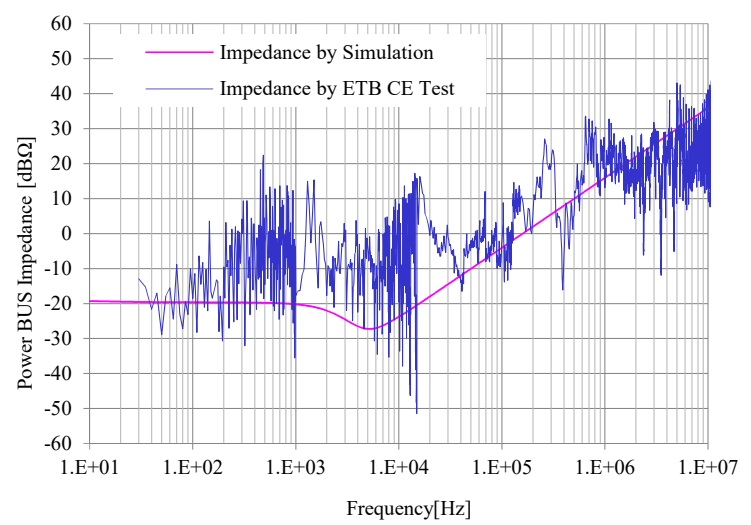

Fig. 18. Comparison of simulated impedance at power star point and calculated impedance using ETB CE test results.

The fluctuation of the measurement result is caused by the test site and test instrument (i.e., EGSEs). The deviations between simulated and measured results could be due to the noises coming from the EGSE and test harness. It can be noted that the impedance calculated using $\mathrm{CE}$ test results and simulation results agree well.

\section{CONCLUSION}

A simplified power bus model has been proposed as a method to evaluate the EMC performance of a spacecraft system. The effectiveness of the proposed simple model was experimentally proven on the ETB platform. A good agreement was achieved between the simulation results and the experimental results for the proposed power bus model. This paper confirms that the power impedance characteristics can be determined with the minimum amount of information, such as the input or output filter of PCDU modules and the battery's mechanical configuration. The proposed model permits the prediction of the power system's influence from the perspective of EMC. 
Therefore, the proposed model can be used to assess the conducted EMC safety margin at the power level via computer simulation. The advantage of the proposed model is that it simplifies the complex power system and that its impedance can be quickly obtained at the early stage of spacecraft system design and development.

This was supported by Chungnam National University, Daejeon, South Korea (No. 2017-1896-01).

\section{REFERENCES}

[1] I. Sharaa, D. N. Aloi, and H. P. Gerl, "EMC model-based test-setup of an electrical system," Progress In Electromagnetics Research B, vol. 11, pp. 133-154, 2009.

[2] G. Spadacini, F. Grassi, and S. A. Pignari, "Modelling and simulation of conducted emissions in the powertrain of electric vehicles," Progress In Electromagnetics Research B, vol. 69, pp. 1-15, 2016.

[3] Z. Jiang, R. A. Dougal, and S. Liu, "Application of VTB in design and testing of satellite electrical power systems," Journal of Power Sources, vol. 122, no. 1, pp. 95-108, 2003.

[4] C. S. Madhusudhana, G. N. V. Prasad, and C. D. Sridhara, "Modelling for evaluating efficiency of spacecraft power systems," in Proceedings of 2014 Power and Energy Systems: Towards Sustainable Energy, Bangalore, India, 2014, pp 64-70.

[5] T. Mesbahi, N. Rizoug, P. Bartholomeus, R. Sadoun, F. Khenfri, and P. Le Moigne, "Dynamic model of li-ion batteries incorporating electrothermal and ageing aspects for electric vehicle applications," IEEE Transactions on Industrial Electronics, vol. 65, no. 2, pp. 1298-1305, 2018.

\section{Tae-Youn Kim}

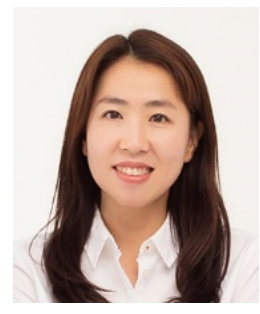

received B.S. and M.S. degrees in Electronic Engineering from Dankook University, Seoul, Korea, in 1999 and 2001, respectively. Since 2001, she has been working as a senior researcher with the Division of Space Environment Test, Korea Aerospace Research Institute, Daejeon, Korea. She is also working toward her Ph.D. degree with the Department of Radio and Information Communications Engineering at Chungnam National University, Daejeon, Korea. Her research interests include electromagnetic compatibility, electrostatic discharge, and RF field analysis.
[6] W. Waag, S. Kabitz, and D. U. Sauer, "Experimental investigation of the lithium-ion battery impedance characteristic at various conditions and aging states and its influence on the application," Applied Energy, vol. 102, pp. 885-897, 2013.

[7] S. L. Wu, H. C. Chen, and S. R. Chou, "Fast estimation of state of charge for lithium-ion batteries," Energies, vol. 7, no. 5, pp. 3438-3452, 2014.

[8] M. S. Tabbakh, F. Morel, R. Mrad, Y. Zaatar, "High frequency battery impedance measurements for EMI prediction," in Proceedings of 2013 IEEE International Symposium on Electromagnetic Compatibility, Denver, CO, 2013, pp. 763767.

[9] P. Pelissou, P. Delannoy, X. Romeuf, and P. Laget, "A high fidelity simulation tool for conducted EMC analysis at system level," in Proceedings of 2012 ESA Workshop on Aerospace $E M C$, Venice, Italy, 2012, pp. 1-5.

[10] D. H. Baert and A. A. K. Vervaet, "A new method for the measurement of the double layer capacitance for the estimation of battery capacity," in Proceedings of the 25th International Telecommunications Energy Conference, Yokohama, Japan, 2003, pp. 733-738.

[11] W. Rubel, "Satellite design, power system," EADS Astrium GmbH, Bremen, Germany, 2006.

[12] C. C. Huang, J.J. Yeh, and C. K. Tseng, "Estimate filter tank fault effects for satellite power system," in Proceedings of the World Congress on Engineering (Vol. 2), London, UK, 2012, pp. pp. 1141-1144.

[13] J.D. Choi, J. C. Koo, C. H. Koo, and E. C. Kim, "Validation of electrical power system using Electrical Test Bed of GEO satellite," in Proceedings of 2007 7th International Conference on Power Electronics, Daegu, South Korea, 2007, pp. 316-319.

\section{Jae-Hyun Lee}

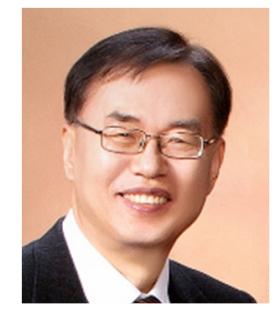

received the B.S. degree in Electronic Engineering from Inha University, Incheon, Korea, in 1982. He received his M.S. and Ph.D. degrees in Electrical and Electronic Engineering from KAIST, Seoul, Korea, in 1985 and 1993, respectively. From 1991 to 2001, he worked as a Senior Researcher with Satellite Research Division, ETRI (Electronics and Telecommunications Research Institute), Daejeon, Korea. Since 2001, he has been a professor in the Department of Radio and Information Communications Engineering, Chungnam National University, Daejeon, Korea. His research interests include electromagnetic compatibility, system and microwave passive components for satellite, microwave signal processing devices. 